\title{
Knowledge Reuse through Categorical Breakdown Analysis: A Method for Collaborative Systems Evaluation
}

\author{
Will Humphries, D. Scott McCrickard, Dennis Neale \\ Center for Human-Computer Interaction, Virginia Tech \\ \{whumphri,mccricks,dneale\}@vt.edu
}

\begin{abstract}
Designing CSCW systems that support the widely varying needs of targeted users is difficult. There is no silver bullet technology that enables users to effectively collaborate with one another in different contexts. We propose a method of collaborative systems evaluation that enables novice evaluators to make insightful observations about the systems they evaluate at a level comparable to experts in certain situations. These observations come in the form of a categorical breakdown analysis of a laboratory study. The quantity and type of breakdowns can then be connected to recommended CSCW tools and features developed and described in the related literature. We conducted a study to explore the results generated when the method was applied by both experts and novices in the field of CSCW. We observed that experts found the method to be usable, and that novices capitalized on the knowledge embodied in the breakdown categories to make categorizations similar to those of experts.
\end{abstract}

\section{Introduction}

When designing collaborative software, it is designers' responsibility to determine not only how users will attempt to collaborate, but also what tools will best support their joint efforts. The growing body of literature related to CSCW provides valuable advice for assessing and designing to meet the collaborative needs of users, but it may be difficult for designers or students who have no prior experience with the design and evaluation of collaborative software to extract relevant knowledge from the literature base.

This paper describes a method designed to help novice evaluators determine what collaborative activities their users are attempting to engage in when adequate system support does not fully exist. Knowledge from CSCW literature is encapsulated within the method, such that in our study the novices performed externally consistent with experts.
Eventually, an interactive guide will allow users to connect the results of their evaluation to documented, peer-reviewed CSCW features which they can consider as potential fixes for the problems highlighted by the evaluation. In this regard, evaluators with little or no knowledge of the CSCW literature base can make use of the guide when determining what collaborative behavior their system needs to support, and what features can be added or modified to better support those behaviors.

\section{Related work}

Evaluating a collaborative system is a complex and difficult undertaking, requiring the use of field methods over extended periods of time [7]. These types of methods can be contrasted with short-term laboratory studies related to the field of HCI that use a range of usability engineering methods to understand single-user systems.

Baker et. al. [1] suggested using heuristic evaluation methods adapted from single-user research on CSCW systems. This approach does provide an efficient and inexpensive option for formative evaluation of groupware systems, but Cockton and Woolrych suggested that such 'discount methods' have significant costs associated with the benefits they provide [5]. These costs include decreased ecological validity as well as a lack of comprehensive testing.

Activity awareness is a concept which builds on prior work done on both social and action awareness. It refers to the ability of the user to have and maintain "awareness of the overall situation, the social expectations and dependencies within their group, and their shared task goals and status" [2]. Activity awareness is knowing what has happened, what is happening, and what will likely happen in the future over extended periods of time.

The concept of activity awareness has recently been clarified with respect to its theory base [3]. A framework is presented which builds on theories of 
interpersonal communication and development. The framework has four facets: common ground, communities of practice, social capital, and human development. These facets are loosely ordered, in that shared understanding within communication (common ground) is necessary for a community to form, and a community will assist its members in gaining trust in one another and learning and developing. We can therefore see that examining how common ground is supported by a groupware system can be very helpful in assessing the potential for that system to maintain activity awareness amongst its user population.

Common ground can be defined as 'what Conversant A knows about what Conversant B knows about what Conversant A knows about...' In other words, common ground is the shared knowledge that we take for granted when conversing [4]. The loss of a shared physical environment hinders common ground. The theory of common ground is applied to activity awareness with a particular emphasis on keeping members in a state of mutual understanding of shared plans, responsibilities, and goals. A group will work more effectively when its members not only know about the shared elements of a task, but also know that their fellow collaborators have this knowledge.

When activity awareness is not maintained, efforts at planning and completing the activity will lead to breakdowns as inconsistencies in different users' interpretations of shared plans and goals become apparent. A well-designed system should keep the user in this state of awareness as some parts of the project plan undergo revision or as the project's direction evolves and changes over time.

Neale provides a model for the evaluation of activity awareness which builds clearly on the concepts of work coupling and common ground [11]. The work proposed in this paper builds on recent work done to develop and validate a new laboratory model for observing complex CSCW phenomena. The model seeks to encourage activity awareness through appropriate work coupling, leading to a degree of common ground that enables all collaborators to have knowledge of the work of each other (see Figure 1). The improvements made to the laboratory model strengthen its connection to the concepts that make up activity awareness.

\section{A laboratory model for evaluating activity awareness}

Researchers developed a laboratory model with the goal of maintaining ecological validity while capitalizing on the benefits of a laboratory study, namely short time span and minimal use of resources [6]. This laboratory model was used to observe activity awareness. Activity awareness was chosen because it is a complex problem central to the effectiveness of CSCW systems [2].

Prior instantiations of the laboratory model consisted of users completing tasks which were designed based on data collected during field work. In the first study conducted, collaborative scenarios were modeled on those commonly observed over the course of a field study exploring inter-classroom collaboration in a long-term school project. Participants in the study were selected from students attending school in grade levels similar to those students observed in the field study. In order to reduce the time required to conduct the experiment, participants were not required to complete all of the work involved in the task (a science project) assigned to them. Instead, they planned the work that would have been eventually completed, and their awareness of these plans was probed over the course of several experimental sessions [6].

In order to introduce ample opportunities to test the strengths and weaknesses of the system, a confederate acted as a collaborator with the study participants. The confederate went through scripted behaviors that were designed to elicit the participants' awareness. It was found that performing the study without the confederate led to a decrease in observed breakdowns, which makes it more difficult for researchers to determine what activities the system is failing to adequately support [9].

\subsection{Breakdown analysis}

The participants' awareness in the studies was primarily assessed using the breakdown analysis technique. This evaluation technique, adopted from Activity Theory, stems from the idea that collaboration is frequently interrupted when two or more users have lost their sense of shared understanding. This loss might be regarding a shared plan, the working environment, conversational common ground, or the context of the shared work. The breakdown occurs when the actions of a participant do not meet the expectations of another collaborator [2].

It is important to note that breakdowns occur in both face-to-face and remotely located collaboration. Breakdowns allow collaborators to realize, work out, and address misconceptions held by the group. In fact, when breakdowns are resolved quickly, they can have a positive impact on social capital [11]. However, fewer breakdowns is more generally desirable and indicates that shared understanding is maximized. The 
evaluator should be aware that breakdowns cannot and should not be eliminated altogether.

Breakdowns in the prior studies were grouped into one of four categories based upon their cause: 1) Situational (environment); 2) Group/User (users and their roles); 3) Task (plans); 4) Tool (software and workspace). These categories allowed researchers to determine which elements of the system were not supporting collaboration particularly well. For example, a high percentage of Group/User breakdowns can indicate that the tools provided for interpersonal communication are not adequate for what the users are attempting to accomplish.

Evaluators with CSCW experience can use this information to think critically about the tool set provided by the system they are evaluating and make recommendations to developers. However, the evaluator must have a significant amount of experience with the different collaborative tools available in order to determine what is leading to more breakdowns and to be able to recommend solutions. We outline a new method of breakdown analysis and categorization intended to ease the prior knowledge requirements imposed on evaluators, while at the same time ensuring the results obtained are relevant to established models of collaboration.

\subsection{Cause-coupling categorization}

Evaluators of collaborative systems are not all formally trained in the field of CSCW. In fact, many developers and managers of CSCW tool development projects will have minimal background experience with the CSCW solutions provided in the literature. Students beginning a study of CSCW may also find themselves in a position where they want to evaluate a system to see what it does well, and what it does not, without having to digest the broad base of CSCW literature. Therefore, we propose a new breakdown categorization method that will allow them to connect the problems that they observe directly with CSCW factors that they should consider.

We will categorize breakdowns based on two dimensions: cause and work coupling level. Historically, work coupling as a conceptual tool has had multiple meanings depending on the discipline where it has been applied. Within the field of CSCW, work coupling is used to define the extent to which the task requires information sharing and communication. The coupling levels used in our proposed model were derived by Neale from existing literature and the field work and featured in his model for evaluating activity awareness[11].

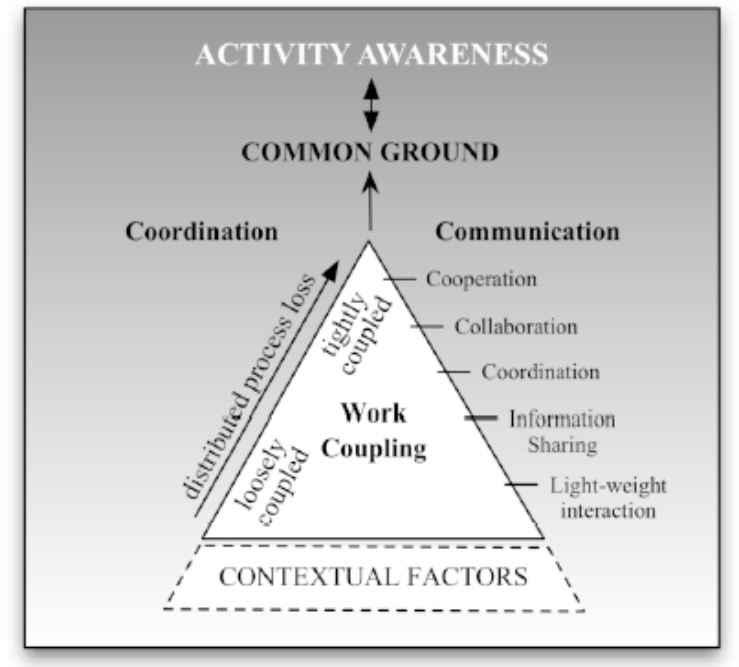

Figure 1: Activity awareness evaluation model

We describe the coupling levels below, ordered from weak coupling to tightly coupled.

1. Lightweight Interaction: Casual social interaction combined with communication about the work. Contextual information is shared as a byproduct of social interaction.

2. Information Sharing: Inform-acknowledge pairs or unidirectional (asynchronous) communications.

3. Coordination: Group members coordinate both activities and communication. Group members coordinate not only work content but also the process involved in carrying it out.

4. Collaboration: Group members work toward a common goal. Collaboration is composed of separate tasks, done by individuals, but with a high degree of interdependence. Goals and tasks are shared, and there is a desire to maintain a high state of shared knowledge

5. Cooperation: Classified by shared goals, common plans, shared tasks, and frequent consultation between members about how to proceed. The team's priorities are of greater concern than individual goals. Work is tightly coupled, ambiguous, and often ill structured. Tasks require high levels of problem solving and constant reassessment of priorities and goals.

Each breakdown will be grouped according to its cause and work coupling level. The result will be a $4 \times 5$ grid of breakdowns. Evaluators will then identify which combinations of coupling level and cause have the highest percentage of breakdowns. This will allow 
evaluators with minimal experience with the field of CSCW to pinpoint problem areas or missing functionality in the systems they evaluate.

\section{Exploring awareness breakdowns}

We conducted a study with the goal of understanding how the evaluation method would be put to use by both experts and novices in the field of CSCW. In particular, we wanted to see whether either or both classes of users could make effective use of the tool. We also wanted to compare the breakdown categorizations generated by novices to those generated by experts, in an effort to assess the consistency of the categorizations generated by evaluators using our framework.

In preparation for the study, we generated awareness breakdowns representative of those other evaluators might find when running a laboratory study of their own. These breakdowns were to be shown to participants in the study to assess the consistency and correctness of their categorization. This also provided us the opportunity to continually test and refine the laboratory method.

Our user population was composed of three graduate students in Computer Science enrolled at Virginia Tech. Participants used the BRIDGE client to complete a collaborative task [10]. The BRIDGE client has five elements (Fig. 2). The timeline tool keeps members apprised of group activity and planning changes. The concept map tool features maps that are used to show relationships between the documents in the workspace. There is also a document editor, a user list, and a chat tool.

Participants worked with a confederate to complete an assignment similar to one they might receive in one of their courses. The task they completed was designed to produce a wide range of coupling levels. The confederate introduced scripted breakdown scenarios during task completion. These scenarios were based on our experience with the type of task they were asked to complete, as well as our familiarity with the BRIDGE client. We also considered the breakdown scenarios used in prior lab studies since those studies also involved participants completing an educational task [6].

Participants clearly were fully engaged in the activity. Our confederate was able to utilize his experience with the work being simulated to provide a realistic collaborative experience for the participants. We identified three breakdowns that are representative of those commonly found using our lab methodology. In one example, the confederate enters some text that the participant is waiting for into a document the participant does not have open. The user doesn't realize the text has been entered, and asks the confederate if s/he can add it to the workspace. Recordings of these breakdowns were used in the study.

\subsection{Expert breakdown categorization}

The purpose of the first phase of our study was to assess the breakdown categorizations derived by users who have a background of designing and evaluating CSCW systems. Our goal was to demonstrate that the distribution of the breakdowns within the cause and work-coupling categories were consistent between several expert evaluators.

Four participants were recruited from faculty and graduate students at Virginia Tech. We screened potential participants to ensure that they had knowledge of CSCW concepts as well as experience applying these concepts to the design and evaluation of collaborative systems. The participants were provided with a brief document describing the process of breakdown analysis, as well as the causes and work coupling levels involved in the breakdowns they would eventually categorize.

Participants were then shown three breakdowns collected in preparation for the study. They were asked to assign a cause and work coupling level to each of these breakdowns. Participants were given control of the recordings and were allowed to review and replay the breakdowns as much as needed. Participants were encouraged to engage in think-aloud as they completed the breakdown analysis.

Three of the four participants identified fitting causes and coupling levels for all of the breakdowns shown to them. One participant felt that one breakdown did not fit into any of the causes outlined by the researchers. However, that user's comments indicated that they agreed with all of the other participants that the breakdown was caused by a fault in the tool.

The comments given by the users indicate that the cause coupling categorization provided is robust enough to handle all of the collaborative breakdowns. In general, their thoughts about breakdowns led them naturally to one or two of the causes and/or coupling levels provided, and subsequently they were able to narrow down the cause to a single choice after reexamining the definitions provided for a category. 


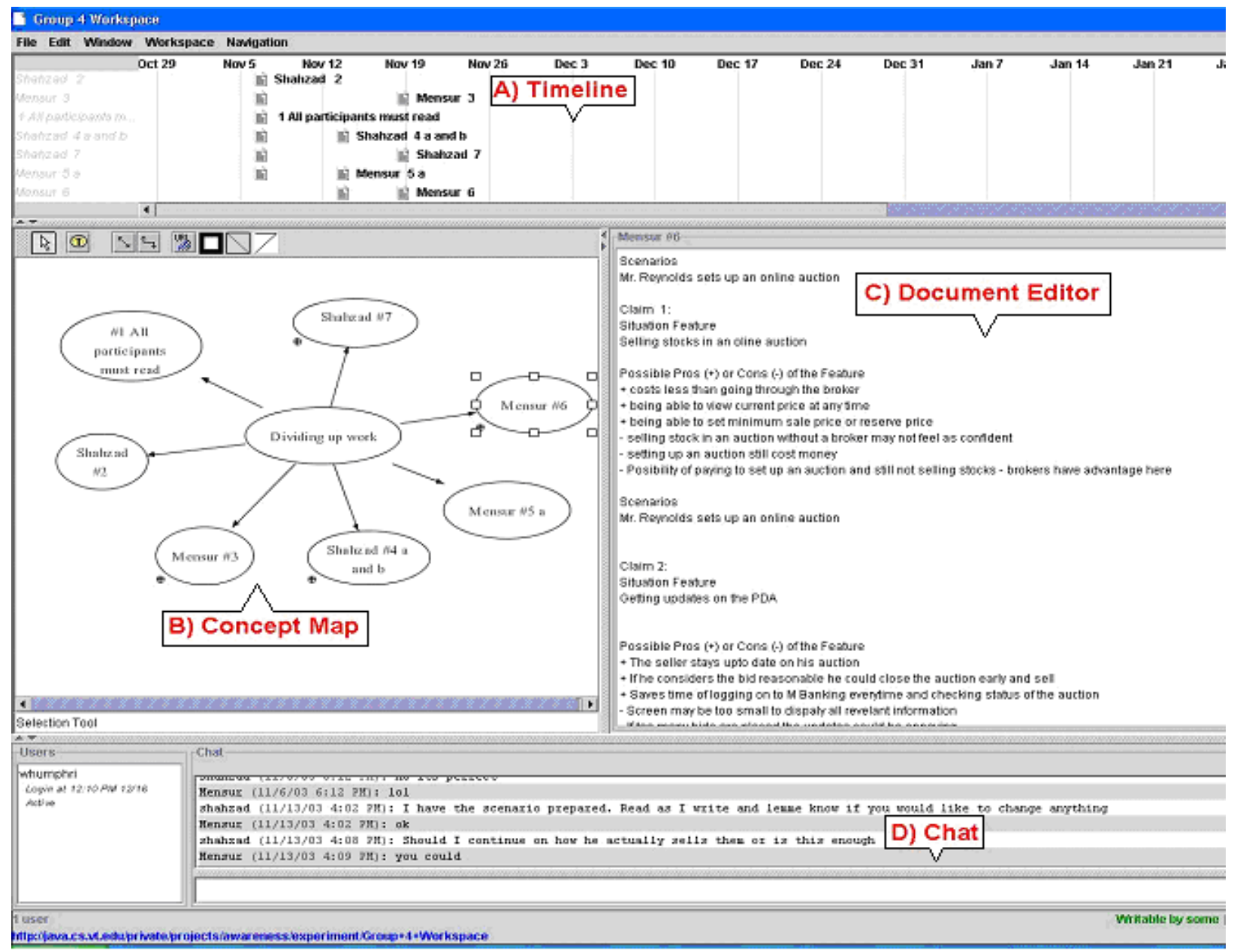

\section{Figure 2: BRIDGE client interface}

We observed greater variation among the coupling level categories chosen by participants. When users collaborate, they do not make discrete movements between different levels of work coupling. However, we feel that asking evaluators to choose a discrete coupling level encourages them to think carefully about the actions and needs of the user, which ideally will lead to better system design.

\subsection{Novice breakdown categorization}

The goal of the second phase of our study was to determine whether novice users could carry out an evaluation of a collaborative tool through the use of our method, and whether their categorizations would be consistent with those generated by experts. If so, these categorizations could be used to recommend improvements to the collaborative system they are evaluating.

Ten participants were recruited for this phase. Participants were undergraduate computer science students enrolled at Virginia Tech. We screened potential participants to ensure that they had little to no knowledge of CSCW concepts. The participants were given the same information that was given to expert evaluators in the phase. They were asked to categorize the same breakdowns.

We found that in every case, the majority of participants (50-80\%) chose the same cause from among the four available options. There was more variance in coupling level selection than in cause selection, but this was expected. In all 3 breakdowns that users categorized, a 2-coupling level range encompassed at least $70 \%$ of the evaluators' responses. The majority of 'outlier' choices were attributed to the evaluators' inexperience with the BRIDGE Client based on the comments provided with choices. Most of the evaluators had only passing familiarity with the tool.

Finally, we compared the categorizations generated by experts to the categorizations generated by novices. We observed that in 2 of the 3 breakdowns 
categorized, the majority of novices chose the same cause as the majority of experts.

\section{Conclusions and future work}

We demonstrated that the laboratory evaluation and breakdown categorization methods described in this paper result in consistent breakdown categorizations when applied by expert users. We also showed that novice evaluators can capitalize on the knowledge embodied in the breakdown categories to make relevant categorizations. This is a resource-efficient way to assess the strengths and weaknesses of a collaborative tool. The results of the evaluation should also provide an opportunity for insight. Evaluators will have an opportunity to think about why their system does or does not support such behaviors as 'loosely coupled planning' or 'tightly coupled communication.' This will allow them to gain a deeper understanding of the potential and limitations of their system. For example, a large number of group/user breakdowns at high work coupling levels indicate that users are attempting to engage in synchronous communication and finding that the tools provided to them are inadequate.

In the future, it may be productive to assess various CSCW features and formally specify which levels of the coupling-level pyramid (Fig. 1) they support. These specifications could be encapsulated in an interactive guide, which would accept as input the observed breakdown causes and coupling levels for a given tool. The guide could then direct novice evaluators towards areas of functionality that their system does not currently offer, or that have been implemented in a way that does not align with the best practices noted in prior work.

\section{Acknowledgements}

We thank Travis Rose for his assistance in carrying out the study. Thanks to Deborah Tatar for her advice and feedback on ideas which led to this work. A special thanks to everyone who participated in our studies.

\section{References}

[1] Baker, K. Heuristic Evaluation of Shared Workspace Groupware based on the Mechanics of Collaboration. Technical Report, Department of Computer Science, University of Calgary, 2002.

[2] Carroll, J.M., Neale, D. C., Isenhour, P. L., Rosson, M. B., McCrickard, D. S. Notification And Awareness:
Synchronizing Task-Oriented Collaborative Activity. International Journal of Human-Computer Studies 58 (5).

[3] Carroll, J.M., Rosson M.B., Convertino, G., Ganoe, C. Awareness and Teamwork in Computer-Supported Collaborations. Interacting with Computers 18 (1). 21-46.

[4] Clark, H.H., Brennan, S. E. Grounding in communication. In Resnick, L.B., Levine, R. M., Teasley, S. D. ed. Perspectives on Socially Shared Cognition, APA, Washington, D.C., 1991, 127-149.

[5] Cockton, G., Woolrych, A. Sale Must End: Should Discount Methods Be Cleared Off HCI's Shelves? interactions, 9 (5). 13-18.

[6] Convertino, G., Neale, D. C., Hobby, L., Carroll, J. M., Rosson, M. B., A Laboratory Method for Studying Activity Awareness. In Proceedings of NordiCHI, (2004), ACM Press, 313-322.

[7] Grudin, J., Why CSCW applications fail: problems in the design and evaluation of organization of organizational interfaces. In Proceedings of CSCW, (1988), 85 - 93.

[8] Humphries, W., McCrickard, D. S., and Neale, D., Knowledge Reuse Through Breakdown Categorization. Technical Report, Department of Computer Science, Virginia Tech, 2008.

[9] Humphries, W., Neale, D. C., McCrickard, D. S., and Carroll, J. M., Laboratory Simulation Methods for Studying Complex Collaborative Tasks. In Proceedings of HFES, (s, 2004), 2451-2455.

[10] Kim, K., Isenhour, P.L., Carroll, J.M., Rosson, M.B., and Dunlap, D.R., TeacherBridge: Knowledge Management in Communities of Practice. In Proceedings of HOIT, (2003). [11] Neale, D.C., Carroll, J. M., Rosson, M.B., Evaluating Computer-Supported Cooperative Work: Models and Frameworks. In Proceedings of CSCW, (2004), 112-121. 